\section{Papillary thyroid cancer and cervical lymph mode metastases - optimal preoperative evaluation}

\author{
George H Sakorafas*
}

Assistant Professor of Surgery, Athens University, Medical School \& F. Chairman, Department of Surgery, 'SAINT SAVVAS' Cancer Hospital, Athens, Greece

\section{Introduction}

Papillary thyroid cancer (PTC) is the most common subtype of thyroid cancer and a highly curable malignancy [1]. However, despite its excellent prognosis, cervical lymph node metastases (CLNMs) are present in a significant percentage of patients with papillary thyroid cancer (PTC) (upto $50 \%-60 \%$ ) [2]. Their presence is associated with an increased risk of local recurrence, while disease-specific mortality remains relatively unaffected. The management of the patients with PTC and CLNMs differs significantly from that of patients without CLNMs. Experience is required from the part of the clinician (endocrinologist/surgeon) performing the preoperative evaluation to organize appropriate diagnostic examinations. Careful interpretation of imaging findings from the part of the radiologist is of crucial importance to recognize CLNMs. Close collaboration with the cytologist is essential to document cytologically their presence (when indicated). Finally, the surgeon performing surgery should be familial not only with the technique of thyroidectomy, but also with the different options of cervical lymph node dissection. He/she should be able to select the appropriate surgical strategy for the individual patient, based on the findings of preoperative evaluation. Both thyroidectomy and cervical lymph node dissection should be performed with minimal morbidity. In this brief report we present currently available data regarding optimal preoperative evaluation of the patient with PTC specifically regarding CLNMs.

\section{Diagnostic approach}

Accurate preoperative identification of CLNMs is the first step in the management of the patient. Currently, preoperative diagnosis of CLNMs is based mainly on preoperative imaging.

\section{Clinical examination}

Despite the central role of modern imaging in diagnosing CLNMs, clinical examination remains the first diagnostic

\author{
More Information \\ *Address for Correspondence: \\ George H Sakorafas, MD, Arkadias 19-21, 115 \\ 26 Athens, Greece, \\ Email: georgesakorafas@yahoo.com \\ Submitted: February 01, 2021 \\ Approved: February 15, 2021 \\ Published: February 16, 2021 \\ How to cite this article: Sakorafas GH. Papillary \\ thyroid cancer and cervical lymph mode \\ metastases - optimal preoperative evaluation. \\ Ann Clin Endocrinol Metabol. 2021; 5: 011-013. \\ DOI: 10.29328/journal.acem.1001018 \\ Copyright: () 2021 Sakorafas GH. This is \\ an open access article distributed under the \\ Creative Commons Attribution License, which \\ permits unrestricted use, distribution, and \\ reproduction in any medium, provided the \\ original work is properly cited. \\ Keywords: Thyroid; Cancer; Thyroidectomy; \\ Metastases; Lymph nodes; Papillary; Lymph \\ node dissection \\ Check for updates \\ OPEN ACCESS
}

examination during preoperative evaluation. Lymph node metastases may be clinically evident, mainly by palpation, when large and relatively superficial, as masses in the neck. However, sensitivity and accuracy are low $(40 \%-50 \%$ and $75 \%$, respectively). Therefore, clinical examination detects lymph node involvement in only $15 \%-30 \%$ of patients [3]. Selection of the optimal management of the patient, therefore, cannot be based on clinical examination alone.

\section{Ultrasonography}

Neck ultrasonography is the 'gold-standard' in the preoperative imaging of the patient with PTC [3]. It is cheap, widely available, easy to perform, without exposing the patient to the risks of radiation. However, ultrasonography is a highly operator-dependent diagnostic method. Ultrasonographic findings suspicious for the presence of CLNMs include:

- Calcifications

- Cystic degeneration

- Loss of echogenic fatty hilum

- Hyperechogenicity

- Round shape

- Abnormal vascularity 
Of these ultrasonographic characteristics, calcification and cystic change have very high specificity and PPV (positive predictive value). These findings are not observed in normal or reactive lymph nodes. Cystic degeneration in lymph node metastases from thyroid cancer is observed in $10 \%-25 \%$ of cases (most commonly in PTC) [4].

Suspicion for malignant involvement of neck lymph node in patients with thyroid cancer should be raised when the criterion involving at least one suspicious finding is present on ultrasound. The accuracy of ultrasonographic identification of metastatic involvement of neck lymph nodes increases by combining these diagnostic criteria.

The role of the radiologist performing preoperative neck ultrasonography is of crucial importance. He/she should be able to recognize suspicious US findings and provide the surgeon with a detailed and accurate description of their location within the anatomic compartments of the neck (ultrasonographic lymphatic mapping).

\section{Cross-sectional imaging (computed tomography [CT]/ magnetic resonance imaging [MRI])}

Cross-sectional imaging is not routinely indicated in the preoperative investigation of the patient with PTC. However, may be required in selected cases, such as [3]:

- Presence of massive, bulky CLNMs

- Suspicion of mediastinal lymph node metastases (in these patients, the role of ultrasonography is very limited, due to the interposition of the sternum)

- Suspicion of extra capsular extension

- Suspicion of invasion of adjacent anatomic structures

Initial concerns regarding the administration of iodinebased contrast during preoperative CT have been proved unfounded, since the contrast material is quickly eliminated by the kidneys within a few weeks. Therefore, radioiodine ablation therapy -if needed- can be administered safely, without unnecessary delays.

Interestingly, recent reports emphasized the role of CT especially regarding the identification of CLNMs within the central compartment of the neck $[5,6]$. It is well known that - especially regarding the central compartment of the neck [level VI] - neck ultrasonography has limited accuracy for the detection of lymph node metastases, even in the hands of the most experienced radiologist, mainly due to the interposition of the two thyroid lobes. In these reports, CT showed higher sensitivity than ultrasound for diagnosing lymph nodes in the central compartment. Unlike ultrasound, in most cases, there were no blind spots for CT. With $1 \mathrm{~mm}$ thin-slice CT images, more lymph nodes can be detected, making it possible to analyze the imaging features of these nodes further.
Moreover, CT multiplanar reconstruction helps to observe small lymph nodes within the central compartment (level VI) from different directions, and it also helps to identify the round shape and other detailed features.

Suspicious malignant lymph nodes (positive findings on CT) are defined as nodes with any of the following typical CT characteristics:

- Longer than $5 \mathrm{~mm}$ in the short diameter

- Cystic change

- Strong enhancement

- Nodular enhancement

- Fusion or clustering

- Calcification

MRI may be indicated under specific circumstances and based on the same criteria, as described above (for CT), mainly to identify lymph node metastases in 'US-difficult' areas (central compartment). MRI is more sensitive than ultrasonography regarding detection of nodal necrosis. Thyroid contour protrusion sign and poorly defined tumor margin in contrast-enhanced imaging could be two important predicted findings for cervical LNMs in PTC [7].

\section{PET/CT scan}

The use of 18-fluoro-2-deoxyglucose (FDG) positron emission tomography (PET) and technetium-99m tetrofosmin (Tc-99 m TF) single photon emission computed tomography (SPECT) has been suggested mainly for the detection of locoregional recurrence after total thyroidectomy and radioiodine-131 therapy. Increasing levels of serum thyroglobulin indicate recurrence and again in these cases initial diagnostic evaluation should be based on neck ultrasonography and CT or MRI. However, PET/CT scan may be indicated in difficult cases, such as after multiple surgeries for locoregional (neck) recurrences. A high FDG avidity of the primary tumor should be viewed as an indication for the presence of CKNM in patients with PTC. Patients with a high pSUVmax should be cautiously assessed for CLNMs and might need a more comprehensive surgical approach [8]. However, PET/CT scan is not indicated in the initial evaluation of the patient with a newly diagnosed PTC (i.e., before initial thyroidectomy).

\section{Fine-needle aspiration (FNA)}

Fine-needle aspiration of suspicious lymph nodes may be required to confirm the diagnosis of lymph node metastases. Cytology of the material obtained, however, from a suspicious lymph node has high false-negative rates $(10 \%-15 \%)$ and relatively low sensitivity $(\sim 80 \%)$. In about $5 \%-10 \%$ of patients the result will be 'non-diagnostic', 
mainly in the presence of cystic degeneration within the lymph node or due to insufficient material in the needle hub. The diagnostic accuracy of FNA is increased by combining cytology examination with thyroglobulin (Tg) measurement in the aspirate (sensitivity, 95\% - 100\%). The diagnostic value for detecting lymph node metastasis of differentiated thyroid cancer is highest when the combination of cytology and Tg-measurement was used and lowest when cytology was used alone (combination of cytology + Tg measurement $>$ Tg -measurement > cytology) [9]. However, Tg levels in the aspirate may be low or even undetectable when the malignant cells within the involved lymph node do not produce $\mathrm{Tg}$ (i.e., poorly differentiated or dedifferentiated or anaplastic thyroid cancer).

When preoperative imaging identifies pathologic or suspicious lymph nodes in the central compartment of the neck, FNA is not indicated, since in this case therapeutic central lymph node dissection is indicated and this can be performed during total thyroidectomy, through the same incision. If, however, preoperative lymphatic mapping suggests the presence of suspicious lymph nodes in the lateral compartments of the neck (levels II to IV), FNA may be indicated for confirm the diagnosis, especially when imaging findings remain questionable [3]. This is an important consideration, since lateral lymph node dissection requires a different surgical strategy and accurate diagnosis is essential in selecting optimal surgical approach.

\section{References}

1. Siegel RL, Miller KD, Fuchs HE, Jemal A. Cancer statistics, 2021. CA Cancer J Clin. 2021; 71: 7-33.

PubMed: https://pubmed.ncbi.nlm.nih.gov/33433946/
2. Grant CS. Recurrence of papillary thyroid cancer after optimized surgery. Gland Surg. 2015; 4: 52-62.

PubMed: https://pubmed.ncbi.nlm.nih.gov/25713780/

3. Haugen BR, Alexander EK, Bible KC, Doherty GM, Mandel SJ, et al 2015 American Thyroid Association Management Guidelines for Adult Patients with Thyroid Nodules and Differentiated Thyroid Cancer. Thyroid. 2016; 26: 1-133.

PubMed: https://pubmed.ncbi.nlm.nih.gov/26462967/

4. Sakorafas GH, Christodoulou S, Lappas C, Safioleas M. Preoperative Detection of Cervical Lymph Node Metastases in Papillary Thyroid Cancer: A Surgical Perspective. Onkologie 2009; 32: 762-766.

5. Kim SK, Woo JW, Park I, Lee JH, Choe JH, et al. Computed TomographyDetected Central Lymph Node Metastasis in Ultrasonography NodeNegative Papillary Thyroid Carcinoma: Is It Really Significant? Ann Surg Oncol. 2017; 24: 442-449.

PubMed: https://pubmed.ncbi.nlm.nih.gov/27624581/

6. Bongers PJ, Verzijl R, Dzingala M, Vriens MR, Yu E, et al. Preoperative Computed Tomography Changes Surgical Management for Clinically Low-Risk Well-Differentiated Thyroid Cancer. Ann Surg Oncol. 2019; 26: 4439-4444.

PubMed: https://pubmed.ncbi.nlm.nih.gov/31583547/

7. Wang H, Liu K, Ren J. Magnetic Resonance Imaging Characteristics of Papillary Thyroid Carcinoma for the Prediction of Cervical Central Compartment Lymph Node Metastasis. J Comput Assist Tomogr. 2019; 43: 963-969.

PubMed: https://pubmed.ncbi.nlm.nih.gov/31162232/

8. Jung JH, Kim CY, Hyun Son S, Kim DH, Jeong SY, et al. Preoperative Prediction of Cervical Lymph Node Metastasis Using Primary Tumor SUVmax on 18F-FDG PET/CT in Patients with Papillary Thyroid Carcinoma. PLoS One. 2015; 10: e0144152.

PubMed: https://pubmed.ncbi.nlm.nih.gov/26636824/

9. Xu Y, Wu D, Wu W, Jiang J, Xi C, et al. Diagnostic value of cytology, thyroglobulin, and combination of them in fine-needle aspiration of metastatic lymph nodes in patients with differentiated thyroid cancer: A systematic review and network meta-analysis. Medicine (Baltimore). 2019; 98: e17859.

PubMed: https://pubmed.ncbi.nlm.nih.gov/31702649/ 